\title{
Mobile Speditionslogistikunterstützung durch integrierte Kommunikationssysteme
}

\author{
Volker Gruhn*, Malte Hülder*, Raschid Ijioui*, Lothar Schöpe** \\ *Universität Leipzig. Lehrstuhl für Angewandte Telematik / e-Business \\ \{gruhn | huelder | ijioui\}@ebus.informatik.uni-leipzig.de \\ **Informatik Centrum Dortmund e.V., Abt. Softwaretechnik \\ lothar.schoepe@icd.de
}

\section{Kurzfassung}

Für Speditionsunternehmen liegt ein erhebliches Potential zur Effizienzsteigerung in der verbesserten Kommunikation zwischen Disponent und Fahrer. Dies bezieht sich insbesondere auf die Übermittlung von Auftrags- und Transportdaten, sowie auf Daten, die zur Auftragsfortschrittskontrolle dienen. In dieser Arbeit werden zunächst einige am Markt verfügbare Systeme vorgestellt und mit ihren Vor- und Nachteilen präsentiert. Im Anschluss wird das Kommunikationssystem vorgestellt, das im Rahmen des Forschungsprojekts „Mobile Spedition im Web (SpiW) ${ }^{1 “}$ entwickelt wird. Dieses Kommunikationssystem soll als Zusatzmodul in eine bestehende IT-Infrastruktur eines Speditionsunternehmens eingebunden werden. Da das Kommunikationssystem eine offene Schnittstelle anbietet, kann es mit verschiedenen Speditionslogistiksystemen integriert werden, jedoch wird hier die Unterstützung der Schnittstelle seitens des Speditionslogistiksystems vorausgesetzt. Durch eine spezielle Workflow-Engine kann das Kommunikationssystem leicht an die unterschiedlichen Arbeitsabläufe in verschiedenen Speditionsunternehmen angepasst werden.

\section{Motivation}

Speditionsunternehmen erzielen einen Wettbewerbsvorsprung, wenn sie durch einen funktionierenden Güterverkehr Transporte schnell und sicher sowie pünktlich und kostengünstig durchführen können. ,Die Zeit wird im Straßengüterverkehr mehr und mehr zu einer kritischen Komponente" [ErWa97]. Dieser Wettbewerbsvorsprung ist umso wichtiger, da durch den Wegfall der Tarifbindung und durch die Globalisierung die Anzahl der Mitbewerber am Markt steigt. Speditionsunternehmen, die durch den Einsatz von mobiler Kommunikation in der Spedi-

\footnotetext{
${ }^{1}$ Gefördert durch das BMBF unter der Kennziffer 01HT0143
} 
tionslogistik die genannten Anforderungen (schnell, sicher, günstig, pünktlich) besser erfüllen können, gewinnen Vertrauen bei ihren Kunden und können sowohl die Kundenbindung erhöhen als auch ihren Marktanteil steigern.

Folgende Probleme können bei der Kommunikation und Kooperation zwis chen den verschiedenen Rollen innerhalb eines Speditionsunternehmens (Fahrer, Disponent, Kunde) auftreten und damit die Erfüllung der Anforderungen behindern [ErKo01]:

- Probleme des Disponenten

- Diskontinuierlicher, mündlicher Informationsaustausch zwischen Disponent und Fahrer führt zu Zeitverzögerungen und Übertragungsfehlern.

- Der Fahrer ist aus Sicht des Unternehmens eine zentrale Informationsquelle jedoch können die bei ihm auflaufenden Informationen bisher nicht ohne Medienbruch in die Speditionssoftware übernommen und damit nicht ohne weiteren manuellen Eingriff nutzbar gemacht werden (z.B. für einen Fuhrparkleitstand, für eine kunden-/prozessbezogene Statistik, für die Preiskalkulation).

- Aufgrund des fehlenden Kenntnisstandes der Disponenten über den Auftragsfortschritt ist eine kurzfristige Umdispositionen nur begrenzt mö g lich.

- Termin- bzw. Soll-Abweichungen des LKW-Güterverkehrs, sofern diese bekannt werden, sind vom Disponenten nur beschränkt steuerbar bzw. zu korrigieren.

- Eine Kalkulation der Betriebs- bzw. Transportkosten ist nur mit zeitlicher Verzögerung zu erreichen.

- Probleme des Fahrers:

- Der Fahrer kann Störungen oft nur fernmündlich ko mmunizieren.

- Der Fahrer hat nur geringen Einfluss auf Tagestouren und Umdispositionen.

- Übermittelte Daten sind zum Teil unvollständig oder falsch.

- Der Disponent ist bei Rückfragen nicht ständig erreichbar.

- Probleme des Kunden:

- Der Auftragsfortschritt ist für den Kunden nicht transparent.

- Verspätungen sind nicht kalkulierbar.

Durch den Wegfall einer standortgebundenen Erfassung und Übertragung von Informationen und der Hinwendung zu einer mobilen Kommunikation, bei der die Endgeräte nicht mehr ortsgebunden sind, sondern entweder selbst portabel sind oder durch eine Verbindung mit einem Fahrzeug eine Portabilität erlangen, können Informationen (Sensorikdaten und Dispositionsdaten) zeitnah übermittelt werden. Diese Informationen und deren Verarbeitung können zur Lösung der Probleme beitragen. Diese Lösungen betreffen dann nicht nur die dispositive Fahrzeug-Einsatzsteuerung, sondern auch die strategische Fuhrparkplanung.

Ein solches Kommunikationssystem sollte idealerweise über generische Schnittstellen mit Speditionslogistikanwendungen integrierbar sein und daher den Vorteil bieten, dass unterschiedlichste Speditionslogistikanwendungen vieler Anbieter integriert werden können. Hierdurch wird eine Trennung zwischen kaufmännischen Dispositions- und Logistiksystemen und Kommunikationssystemen 
erreicht. Unternehmen sind dann nicht gezwungen, monolithische Softwaresysteme (z.B. Flottenmanagementsysteme [JuLa01]) mit einem umfangreichen Funktionsumfang zu kaufen und einzusetzen, nur um die Kommunikationsfunktionalität zu nutzen. Ebenso wird durch diese Trennung der Investionsschutz für Softwaresysteme unterstützt, d.h. existierende Softwaresysteme können weiterhin genutzt werden und der Datenbestand dieser Systeme braucht nicht migriert zu werden. Hierdurch wird die Möglichkeit - gerade auch für kleine und mittelständische Unternehmen (KMU) - geschaffen, sich durch den Einsatz neuer Softwaresysteme am Markt zu behaupten.

Durch die Trennung von Kommunikationssystem und Speditionslogistiksystem ist die getrennte Wartung und Weiterentwicklung der beiden Systeme möglich. So kann das Kommunikationssystem mit der Verfügbarkeit neuer Standards (z.B. HSCSD, GPRS, UMTS) im Funktionsumfang erweitert werden (z.B. Übertragung von Fotos und Videos, Verbesserung der Benutzerinteraktion), ohne dass notwendigerweise das Speditionslogistiksystem überarbeitet werden muss.

\section{Stand der Forschung und der Technik}

Die Erarbeitung von Möglichkeiten zur Unterstützung der Speditionslogistik ist bereits schon seit einiger Zeit Gegenstand von Forschungsprojekten bei Instituten und Hochschulen und ebenso Aufgabe von Softwareentwicklungen bei Unternehmen.

An der Universität Koblenz wurde im Mai 2000 das Projekt FlottHit begonnen [Flott00]. Ziel dieses Projektes ist die Steigerung der Effizienz und die Optimierung der Logistikprozesse in einem Speditionsunternehmen, die durch den verstärkten Einsatz von Telematik erreicht werden soll.

Die Universität Bremen hat zum April 2001 das Projekt WapLog begonnen [ErKo01], [SiEK03]. Das Projekt hat die Erarbeitung eines Konzeptes zum Ziel, um die Speditionsflotten optimal mit Handy und Internet zu steuern. Dies soll mit Hilfe einer WAP-basierten Informations- und Kommunikationstechnologie realisiert werden.

Die Optimierung und Verwaltung der Fracht und die Kommunikation mit den Fahrern ist mit der heutigen Kommunikationstechnik zwar möglich, aber noch gibt es wenige Systeme, die alle diese Möglichkeiten ohne Medienbruch integrieren. Im Folgenden werden einige Systeme beschrieben, die die Verwaltung und Steuerung eines Fuhrparks unterstützen und die marktbestimmend verfügbar sind [JuLa01]:

- WEBfleet [Data03] ist ein Service für die Ortung und Verfolgung von Fahrzeugen. Dafür werden Techniken wie GPS-Satellitenortung, mobile Datenkommunikation, digitale Straßenkarten und Internet genutzt. Es lassen sich Positionsdaten, Textnachrichten, Meldungen über Betriebs- oder Ladezustände, Identifikationsnummern eingescannter Pakete und weitere Informationen 
versenden, verwalten und auf einer digitalen Straßenkarte bis hin zum detaillierten Stadtplan darstellen.

Dieses Softwaresystem läuft ausschließlich auf den Servern des Anbieters und umfassende Schnittstellen zum bereits vorhandenen Speditionslogistiksystem eines Speditionsunternehmens werden nicht angeboten.

- Eine weitere Lösung zur Optimierung der Fuhrparkmanagementprozesse ist das System der Firma Transwatch [Tran03]. Durch dieses System werden bestimmte Endgeräte wie z.B. Navigationssysteme, Mobiltelefone und PDAs verschiedener Hersteller verwendet. Dieses System bietet ebenfalls Dienste zur Verwa 1tung von Fahrzeugen, Ortung, Nachrichtenübermittlung, sowie zur Auftragsabwicklung. Ein Unterschied zu Webfleet liegt in der Nutzung des Passo-Dienstes [Voda01], welcher das System um wichtige aktuelle Verkehrsinformationen bereichert. Aufgrund dieses Dienstes kann Transwatch jedoch nicht international betrieben we rden.

- Das System FleetBoard [DaCh03] ist ein telematikgestützter Internetdienst für das Verwalten und Managen der Flotte eines Unternehmens. Es benutzt wie das System Webfleet das Internet als Übertragungsmedium und ermöglicht so einen räumlich unbegrenzten Zugriff auf Informationen. Die Dienste dieses Systems sind Analyse der Fahrweise, Tourenplanungs - und Auftragsübermittlung sowie Sendungsverfolgung.

Die Fahrzeuge benutzen eine GPS- und eine GSM-Komponente zur Erfassung und Übertragung von Informationen (Dispositions- und Positionsdaten). Die Darstellung von Fahrzeugpositionen erfolgt, ähnlich den anderen Systemen, auf einer digitalen Landkarte auf dem Display. Das System übermittelt die Daten an einen Server in der FleetBoard-Zentrale. Von dort aus sind die Auswertungen und Daten über das Internet abrufbar - vorausgesetzt, der Anwender hat eine entsprechende, von FleetBoard vergebene, Zugangsberechtigung. Das System läuft ausschließlich auf den Servern des Anbieters. Die Integration des Servers in die Infrastruktur eines Unternehmens wird nicht angeboten.

- Das Softwaresystem DORIS [Dori03], benutzt ebenfalls das Internet als Übertragungsmedium. Das System ist international einsetzbar und die Fahrzeuge bzw. Fahrer sind mit PDAs ausgestattet. Die Positionsbestimmungen werden über das GPS-System ermöglicht. Das Senden und Empfangen von Informationen geschieht durch den in GSM-Netzen angebotenen SMS-Dienst. Die Offenheit des Systems erlaubt im Gegensatz zu anderen Systemen die Integration in eine bestehende Infrastruktur des Unternehmens. Das System an sich ist modular aufgebaut, wodurch man die Möglichkeit hat, jederzeit neue Bausteine zu integrieren bzw. zu ergänzen. Die Software, der Daten- und Kommunikationsfluss, Transaktionssteueranweisungen und die Systembetreuung liegen zentral bei der Domino EDV-Kommunikation $\mathrm{GmbH}$.

All diese Systeme haben aber die eine oder andere Schwäche. So werden WEBfleet, Transwatch und FleetBoard zentral auf einem Server des jeweiligen 
Dienstleisters betrieben. Dadurch werden relevante Firmendaten bei einem Drittanbieter verwaltet und die Spedition wird abhängig von der Verfügbarkeit und Sicherheit des angebotenen Dienstes. Beim Doris -System besteht zwar die Möglichkeit, das System in die eigene Infrastruktur zu integrieren, jedoch ersetzt Doris die bisher eingesetzte Speditionslogistikanwendung. Die Disponenten müssen daher auf komplett neuen Systemen geschult werden, was weitere Kosten erzeugt. Bei allen Systemen müssen Daten aus der bestehenden Speditionslogistikanwendung migriert werden, ein zeitaufwändiger und fehlerträchtiger Vorgang.

\section{Anwendungsszenario}

Im Rahmen eines Güterverkehrs wird ein Speditionsunternehmen beauftragt, bei einem Versender eine Anzahl von Ladungen abzuholen und auszuliefern.

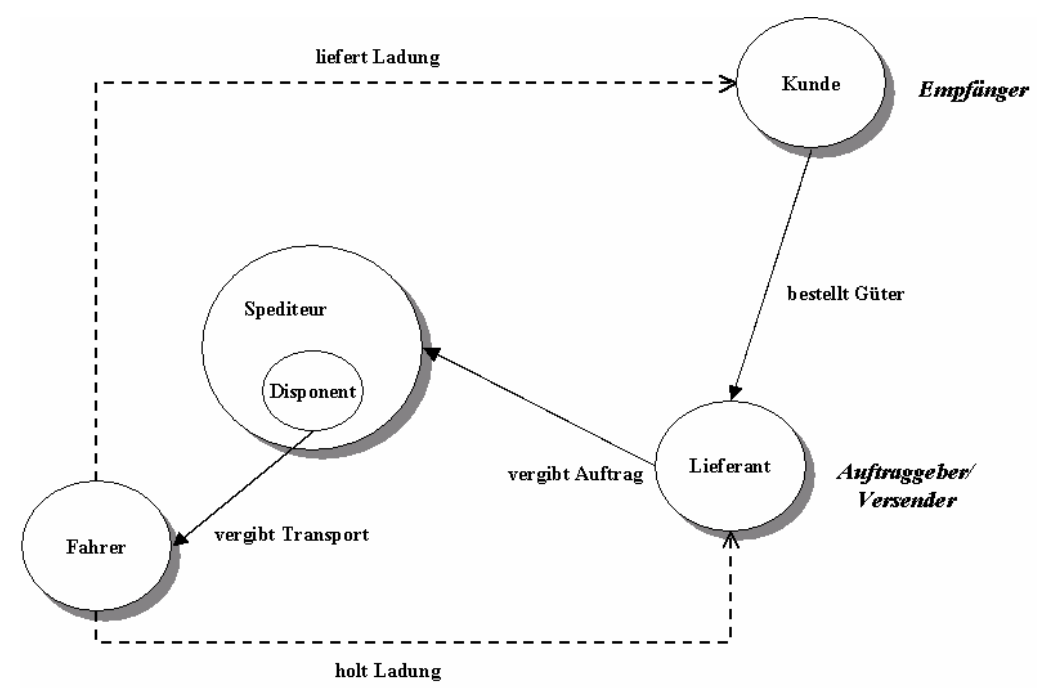

Abbildung 1: Rollen im Güterverkehr

Das Speditionsunternehmen vergibt einen Transport an einen Fahrer - angestellt oder Subunternehmer -, der die Ladungen bei dem Versender abholt und nacheinander an die Empfänger ausliefert (vgl. Abbildung 1: Rollen im Güterverkehr).

Durch dieses Szenario wird ausschließlich der Ganzladungsverkehr und Teilladungsverkehr (z.B. Transport von Stahl und Stahlerzeugnissen) dargestellt. Der Ganz- und Teilladungsverkehr ist eine spezielle Variante des Güterverkehrs und unterscheidet sich im Vergleich zu den KEP-Diensten (Kurier-, Express- und Paket-Diensten) dadurch, dass kein Vor- oder Nachlauf stattfindet und dass die Kunden (Versender, Empfänger) selten Privatpersonen, sondern in der Regel Unternehmen sind. 
Zur Unterstützung der Kommunikation und Kooperation zwischen den beteiligten Rollen in diesem Szenario werden technische Hilfsmittel und Softwaresysteme eingesetzt. Das Speditionsunternehmen setzt Speditionslogistikanwendungen ein, um den Disponenten bei der Disposition von Aufträgen zu unterstützen. Die Kooperation und Kommunikation mit dem Fahrer erfolgt in der Regel mündlich per Telefon/Mobiltelefon oder schriftlich mittels Dokumenten (Tourenpläne, Lieferscheine, Ladelisten). Die Kommunikation zwischen dem Disponenten und den Kunden (Empfänger/Lieferanten) erfolgt telefonisch oder schriftlich per Fax oder mittels elektronischen Datenaustausches (EDI) über eine Schnittstelle der Speditionslogistikanwendung.

Bei der ausschließlichen Verwendung dieser technischen Hilfsmittel können die in der Einleitung genannten Probleme auftreten.

Der Einsatz eines Kommunikationssystems als Ergänzung zu einer Speditionslogistikanwendung soll bei der Problemlösung helfen.

Die Benutzer eines solchen Kommunikationssystems sind hauptsächlich die Disponenten und die Fahrer eines Speditionsunternehmens. Durch die Integration mit einer Speditionslogistikanwendung und die mögliche Erweiterung der Kundenschnittstelle des Speditionslogistiksystems können auch die Kunden das Kommunikationssystem eingeschränkt nutzen. So können über die entsprechenden Schnittstellen (EDI) beispielsweise Informationen zu Transportaufträgen in das Speditionslogistiksystem eingespielt werden, die nach der Disposition an den Fahrer übermittelt werden. Andererseits kann die Übermittlung von Transportstatus und Position des Fahrzeugs für eine Tracking und Tracing Anwendung genutzt werden.

Es werden hier daher im Wesentlichen zwei Arten von Benutzern unterschieden, die auch verschiedene Geräte verwenden. Während der Fahrer eines Speditionsunternehmens ein mobiles Endgerät (PDA - Personal Digital Assistant) nutzt, nutzt der Disponent ein stationäres Endgerät ( $P C$ - Personal Computer $)$.

\section{Architektur}

Die Systemarchitektur des Kommunikationssystems besteht aus drei Ko mponenten: mobilen Endgeräten, stationären Endgeräten und einem Anwendungsserver. Die mobilen Endgeräte nutzen ein leiterungebundenes Medium (GSM, GPRS, $H S C S D, U M T S)$ zur Kommunikation mit einem Anwendungsserver, während durch das stationäre Endgerät ein leitergebundenes Medium (Ethernet, FastEthernet) zur Kommunikation mit dem Anwendungsserver verwendet wird.

Die Softwarearchitektur des Kommunikationssystems folgt dem Client/ServerKonzept [Lewa98]. Durch einen Anwendungsserver wird die Geschäftslogik zur Bearbeitung von Geschäftsobjekten [BaGe98] bereitgestellt. Zur Umsetzung, Unterstützung und Ausführung dieser fachlichen Geschäftslogik bedient sich der Anwendungsserver weiterer Serverkomponenten: einem Workflow-Server, einem Kommunikations-Server und einem Datenbank-Server (vgl. Abbildung 2: Architektur des Kommunikationssystems). Durch die Clients werden die vom Server 
entsprechend der fachlichen Geschäftslogik angebotenen Dienste genutzt, um Daten zielgerichtet einem Benutzer zur Bearbeitung zur Ve rfügung zu stellen.

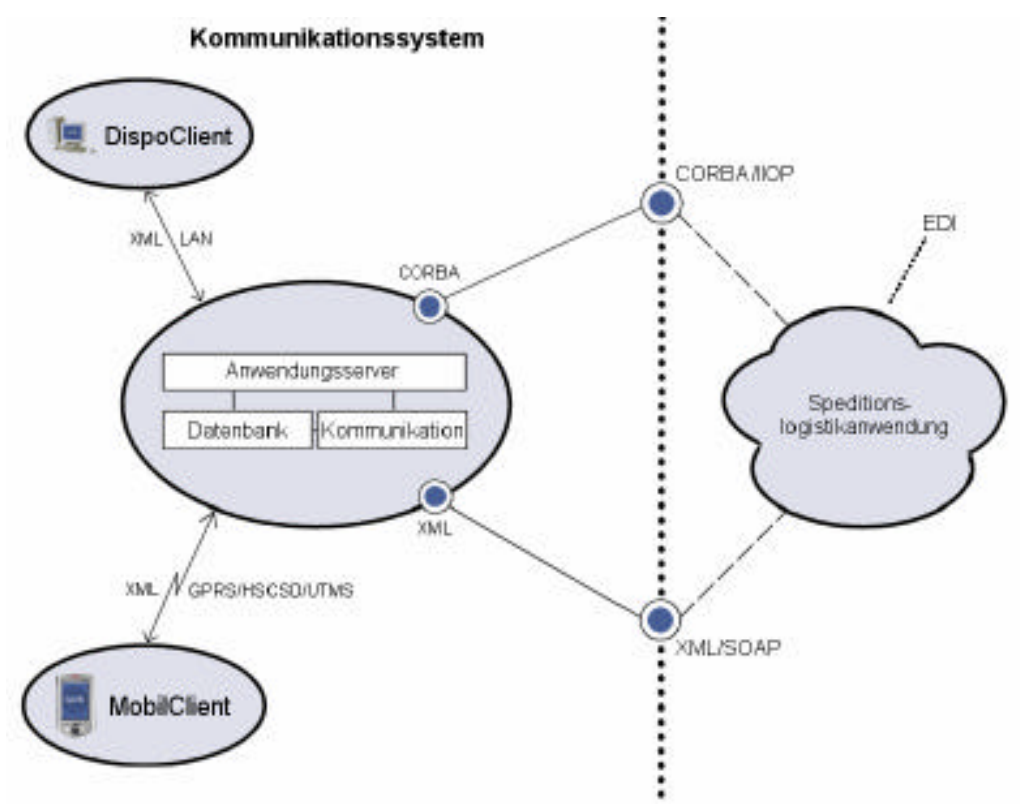

Abbildung 2: Architektur des Kommunikationssystems

Die Art der Daten, die durch die verschiedenen Clients zur Bearbeitung zur Verfügung gestellt werden, ist unterschiedlich. Nicht jeder Benutzer benötigt aufgrund seiner Rolle die gleichen Daten. Während der Fahrerim Wesentlichen nur Transportdaten benötigt, ist der Disponent neben den Transportdaten auch an den Dispositionsdaten interessiert. Durch die verschiedenen Clients werden in Abhängigkeit von der Art des Endgeräts und dessen technischen Möglichkeiten (z.B. Größe des Displays), nicht nur die Daten unterschiedlich dargestellt, sondern auch die Menge der dargestellten Daten begrenzt. Ebenso ist die Art der Benutzungsführung unterschiedlich (z.B. durch vordefinierte Tasten beim PDA oder Zeigersteuerung durch eine Maus beim PC).

Wenn die Geschäftslogik nicht nur durch einen Anwendungsserver bereitgestellt wird, sondern Teile der Geschäftslogik auf einen Client verlagert werden, werden Clients als Thick-Clients [Lewa98] bezeichnet. Dagegen verfügen ThinClients (z.B. Web-Browser) über keine eigene Geschäftslogik, sondern nutzen ausschließlich die von einem Anwendungsserver bereitgestellten Dienste.

Obwohl Thin-Clients in der Regel weniger Ressourcen beanspruchen und sich daher besonders für Endgeräte mit geringer Prozessorleistung und Speicheraustattung eignen, kommen auf den mobilen Endgeräten Thick-Clients zur Anwendung. Dies liegt in der Tatsache begründet, dass nicht immer sichergestellt werden kann, dass die Kommunikationsverbindung über ein leiterungebundenes Medium zwischen mobilen Endgeräten und dem Anwendungsserver verfügbar ist. Um jedoch 
den Anforderungen, die aus den in der Einleitung genannten Problemen resultieren, gerecht zu werden, muss ein Teil der Geschäftslogik auch weiterhin ausgeführt werden können, wenn die Kommunikationsverbindung zeitweise unterbrochen ist. Nach Wiederherstellung der Kommunikationsverbindung muss dann eine Synchronisation stattfinden.

Die Wahrscheinlichkeit, dass eine Kommunikationsverbindung über ein leitergebundenes Medium in einem Local Area Network (LAN) über längere Zeit ausfällt, ist gering, sodass diese Gründe bei stationären Endgeräten nicht zum Tragen kommen. Auf Seiten des stationären Clients kommt jedoch ebenfalls ein ThickClient zur Anwendung, der in der Form einer Speditionslogistiksoftware die gesamte Geschäftslogik zur Disposition und zur Anzeige und Bearbeitung der verschiedenen Daten enthält. Über die weiteren Vor- und Nachteile von Thin- und Thick-Clients sei auf [Lewa98] und [OrHE96] verwiesen.

Das Kommunikationssystem stellt Schnittstellen zum Austausch von Daten mit Speditionslogistikanwendungen zur Verfügung.

Für den Austausch von Daten zwischen den Clients und dem Anwendungsserver wird die Struktur der Daten durch jeweils eine Document Type Definition (DTD) [Tolk99], [BöU199] beschrieben und anschließend entsprechend des XML Formats ausgetauscht. Dazu werden zunächst die Attribute der Objekte in XML Daten transformiert und dann über die Kommunikationsverbindung übertragen. Bei der Übertragung können noch zusätzliche Kompressions- und Verschlüsselungsverfahren eingesetzt werden. Auf Seiten des Empfängers wird nun das XMLDokument durchsucht (Parsing) und aus der Struktur und dem Inhalt des Dokuments werden Kopien der Objekte wiederhergestellt.

Dieses Vorgehen ist erforderlich, da bei der Realisierung des Anwendungsservers und der Clients ein Komponentenmodell [GrTh00], [Szyp98] zusammen mit einer objektorientierten Programmiersprache verwendet wird. So muss auch bei späterer Ergänzung des Systems nicht die gesamte Datenübertragung neu entwickelt werden, da lediglich die neuen Klassen auf den Systemen installiert und die $D T D$ erweitert werden muss. Der Parser hingegen kann gemäß des objektorientierten Paradigmas die Methoden der einzelnen Objekte benutzen, um die Umwandlung der Objektdaten in XML-Code und zurück zu veranlassen.

\section{Workflow-Unterstützung}

Die fachlichen Geschäftsprozesse, die durch das Kommunikationssystem unterstützt werden sollen, werden durch sog. Workflows beschrieben (vgl. Abbildung 3: Fachliche Geschäftsprozesse). Durch jeden Workflow wird genau ein fachlicher Geschäftsprozess (z.B. Verweigerung der Warenannahme durch einen Kunden) mit seinen Bedingungen und Entscheidungen beschrieben. Fachliche Geschäftsprozesse können zusammenhängen, d.h. sie können sich initiieren oder bedingen (z.B. die Erfassung einer Annahmeverweigerung durch einen Fahrer bei einem Kunden löst durch eine Änderung des Auftragsstatus eine Benachrichtigung an 
den Disponenten aus, damit die Erstellung eines Retourenauftrags durchgeführt werden kann)

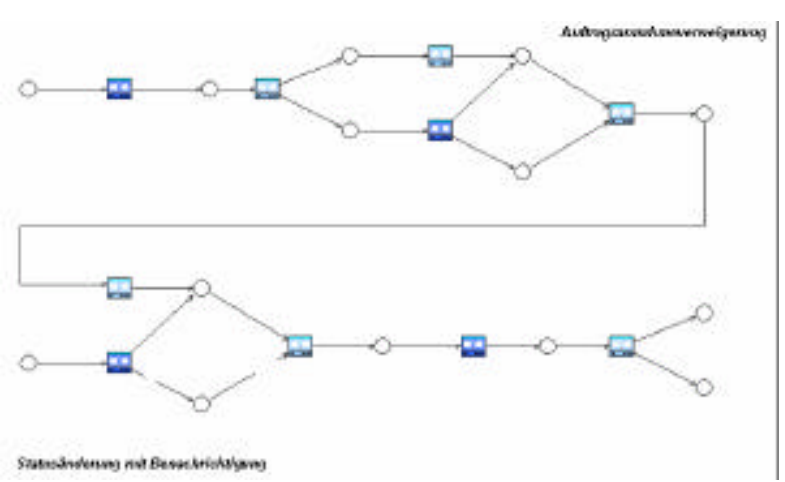

Abbildung 3: Fachliche Geschäftsprozesse

Ein Workflow selbst besteht aus einer Menge von einzelnen Tätigkeiten, die wiederum einfach oder komplex sein können. Diese Tätigkeiten eines Workflows werden entweder sequentiell bzw. parallel oder alternativ zueinander durchgeführt.

Für jedes Teilsystem der fachlichen Architektur (vgl. Abbildung 4: Teilsysteme des Kommunikationssystems ) werden ein oder mehrere Workflows beschrieben, durch die die fachlichen Daten - d.h. die Instanzen der Klassen innerhalb eines Teilsystems - erstellt, bearbeitet und angezeigt werden können.

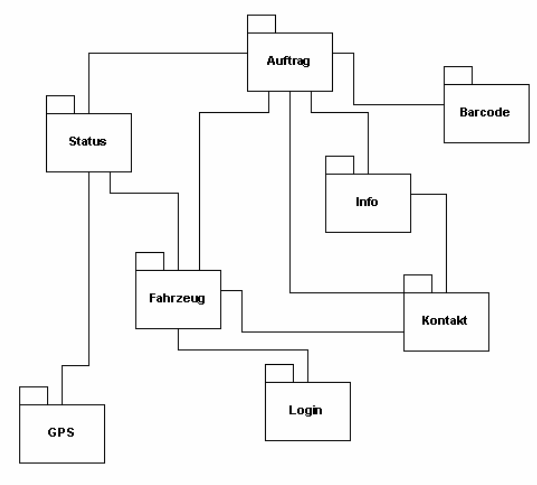

Abbildung 4: Teilsysteme des Kommunikationssystems

Entsprechend der Abhängigkeiten zwischen Teilsystemen können verschiedene fachliche Workflows unterschiedlicher Teilsysteme ebenfalls voneinander abhängig sein. Diese Abhängigkeiten sind nicht immer fest, sondern können sich erst nach der Modifikation oder Erstellung von Daten ergeben (Entscheidung), u.U. auch erst nach dem Lesen von Daten.

Jede Klasse eines Teilsystems enthält eine Display-Methode, mit der die sich Daten dieser Klasse - unter Berücksichtigung eines Style-Guides und entspre- 
chender Benutzungsführung - darstellen. Hierdurch wird sichergestellt, dass die Darstellung von Daten auf dem mobilen Endgerät immer in der gleichen Art und Weise erfolgt und dass die Benutzerführung zur Bearbeitung der Daten ebenfalls immer gleich erfolgt.

In Ergänzung zum globalen Workflow, durch den fachliche Geschäftsprozesse beschrieben werden, beschreibt ein lokaler Workflow den Ablauf von Methoden innerhalb einer Klasse. Dieser lokale Workflow wird durch Realisierung der Display-Methode einer Klasse bestimmt.

Jede Klasse eines Teilsystems wird als Komponente realisiert und implementiert. Bei dieser Realisierung werden die Schnittstellen für die Komponente festgelegt. Durch die Komponente werden die Daten gekapselt und durch Verwendung einer Datenhaltung dauerhaft gespeichert. Die Realisierung der Komponenten erfolgt in einer objektorientierten Programmiersprache (JAVA, C\#).

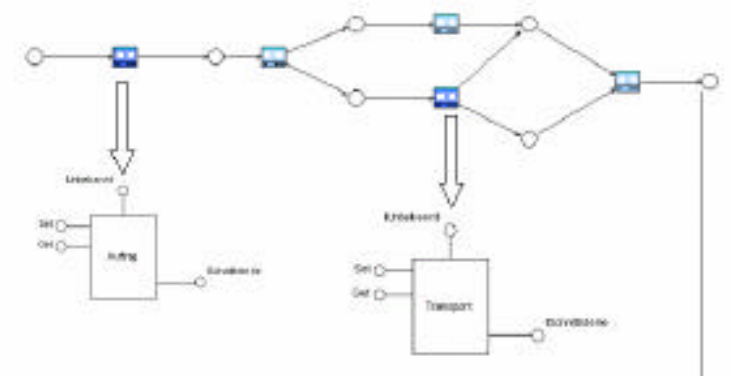

Abbildung 5: Realisierte und zugeordnete Komponenten

Die Komponenten werden den Tätigkeiten eines Workflows zugeordnet (vgl. Abbildung 5: Realisierte und zugeordnete Komponenten). In der Regel sollte Tätigkeiten jeweils nur eine Komponente zugeordnet werden und nur in Ausnahmefällen kann eine Zuordnung von mehreren Komponenten erfolgen. Eine derart ige komplexe Zuordnung soll aber nur dann stattfinden, wenn eine fachliche Restrukturierung des Workflows nicht möglich ist. Der Workflow zwischen den zugeordneten Komponenten muss dann zusätzlich zu dem globalen Workflow und den einzelnen lokalen Workflows explizit beschrieben werden.

Wenn bei der Durchführung einer Tätigkeit eine Entscheidung - auf der Basis von Daten - getroffen werden muss, die den weiteren Ablauf eines Workflows bestimmt, muss für die entsprechende Tätigkeit eine Entscheidungstabelle angegeben werden (vgl. Abbildung 6: Entscheidungstabellen für Tätigkeiten). 


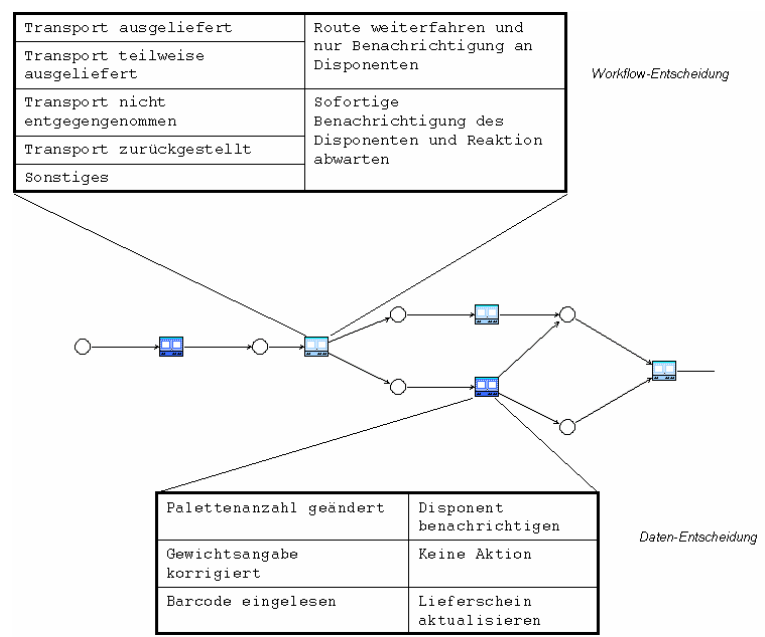

Abbildung 6: Entscheidungstabellen für Tätigkeiten

Die Steuerung der Workflows erfolgt durch einen Workflowserver, der Teil des Anwendungsservers ist. Da Bestandteile eines fachlichen Geschäftsprozesses verteilt - sowohl auf den Clients als auch auf dem Anwendungsserver - ausgeführt werden, muss der Workflowserver auch die verteilte Ausführung von Komponenten unterstützen und die Konsistenz und Integrität sicherstellen. Diese verteilte Ausführung kann zentral oder dezentral gelöst werden. Unter Berücksichtigung der leiterungebundenen Kommunikation zwischen den mobilen Clients und dem Anwendungsserver und den daraus resultierenden Problemen (Verbindungsabbruch, Verbindungsverfügbarkeit, etc.), bietet sich eine dezentrale Lösung an, bei der sowohl auf den mobilen Clients als auch auf dem Anwendungsserver jeweils ein Workflowserver eingesetzt wird, die jedoch in der Mächtigkeit ihrer Funktionalität verschiedenen sind (vgl. Thin-/Thick-Clients). Die Frage, ob Ko mponenten zur Ausführung auf den mobilen Clients dynamisch nachgeladen werden können / müssen oder ob die Komponenten auf den Clients dauerhaft verfügbar sein sollten, wird durch eingesetzte Kommunikationstechnik (HSCSD, GPRS, EDGE, UMTS) - die das Unternehmen vorgibt - bestimmt.

\section{Fazit und Ausblick}

Eine elektronische Unterstützung der Transportdurchführung ist aus informationstechnischer Sicht bisher nur schwach mit Speditionslogistikanwendungen integriert. Eine Kommunikation während der Transportdurchführung bzw. bei seiner Nachbereitung findet per Papier mittels Formularen oder per Telefon statt, was wiederum eine nachträgliche elektronische Erfassung von Informationen bedingt. Diese nachträgliche Erfassung ist fehlerbehaftet. Der Disponent ist während der 
Transportdurchführung nicht ausreichend über den Fortschritt informiert und muss sich bei Bedarf erforderliche Informationen aktiv beschaffen.

Es besteht daher einerseits der Bedarf an einer bidirektionalen Kommunikation zum zeitnahen Austausch von Informationen für die Transportdurchführung und Transportplanung zwischen den Fahrern und dem Disponenten und anderseits der Bedarf an einer unidirektionalen Kommunikation zwischen den mobilen Endgeräten und anderen nachgelagerten Softwaresystemen (Finanzbuchhaltung, Fuhrparkplanung, etc.) zum Austausch von Informationen (Rechnungsdaten, Sensorikdaten, etc.) über ein stabiles leiterungebundenes Medium.

Der Vergleich von am Markt befindlichen Systemen hat gezeigt, dass hier vielfach an den Bedürfnissen kleiner und mittelständischer Speditionsunternehmen vorbei geplant wurde. Diese Betriebe haben oftmals nicht die finanziellen Mittel und nicht das Interesse, in eine komplett neue Software zu investieren. Mitarbeiter müssten zusätzlich geschult werden und nach wie vor ist der Markt für Speditionssoftware so zersplittert, dass kaum jemand guten Gewissens sagen kann, welche Systeme sich langfristig halten und dadurch Investitionssicherheit garantieren werden. Systeme, die auf Application Service Providing Konzepten beruhen, bauen noch eine zusätzliche Hürde auf, da hier vitale Unternehmensdaten wie z.B. Kundenstammdaten und Fuhrparkdaten aus dem Unternehmen an eine dritte Stelle ausgelagert werden. Unternehmen werden somit von der Verfügbarkeit und Datensicherheit bei dieser dritten Stelle abhängig.

Das vom BMBF geförderte Verbundprojekt Mobile Spedition im Web (SpiW) verfolgt daher zwei wesentliche Ziele bei der Entwicklung eines Kommunikationssystems, welche durch das Projektkonsortium definiert wurden:

- Integration mit Speditionslogistikanwendungen,

- Nutzung neuer Telematik- und Kommunikationstechniken.

Im Rahmen dieses Forschungsprojekts ist es aber nicht möglich, diese Anforderungen in Gänze umzusetzen, insbesondere bei der Integration von Speditionslogistikanwendungen kann zwar eine offene Schnittstelle angeboten werden, die Unterstützung dieser Schnittstelle muss aber von den Entwicklern der Speditionslogistikanwendungen erfolgen.

Die komponentenbasierte Architektur des Kommunikationssystems ermöglicht es, dass das System auch später um die Erfassung und Übertragung neuer Daten erweitert werden kann. Hier sind zum einen Foto- und Videodaten denkbar, zum anderen aber auch die Vernetzung mit Boardsensoren und Mautgeräten.

Trotz des zu erwartenden Nutzens eines integrierten Kommunikationssystems für die Speditionslogistik spielen die Kosten für die Anschaffung und - wichtiger noch - für den Betrieb eine zentrale Rolle bei der Konzeption und Realisierung.

Durch die industriellen Partner in dem Verbundprojekt soll sichergestellt werden, dass einerseits diese Aspekte bei der Konzeption und Entwicklung des Kommunikationssystems auch kontinuierlich berïcksichtigt werden und andererseits eine weitere Nutzung und Vermarktung des Systems bei Bedarf über das Ende des Projekts hinaus gewährleistet werden kann. 


\section{Literaturverzeichnis}

[BaGe98] Baker, S., R. Geraghty (1998). Java For Business Objects. In: Carmichel, Andy (ed.): Developing Business Objects, SIGS, Cambridge University Press, pp. 225-237

[BöU199] Böhnlein, U., A. Ulbrich vom Ende (1999). XML - Extensible Markup Language. In: Wirtschaftsinformatik, Band 41, Heft 3, Vieweg Verlag Wiesbaden, pp. 275-277

[DaCh03] DaimlerChrysler Services FleetBoard GmbH (2003). Fleetboard - Flottenmanagement mit IQ. http://www.fleetboard.info/, Stand: 09.02.2003

[Data03] Webfleet-Datafactory AG (2003). Webfleet-Fahrzeug- und Personenortung im Internet. http://www.webfleet.de, Stand: 10.02.2003

[Dori03] DORIS Domino EDV-Kommunikation (2003). Disponenten - Online - Regie und Informationssystem. http://www.mylogistics.net/de/katalog/anbieter/key/ anbieter1473/jsp, Stand: 10.02.2003

[ErKo01] Erkens, E., H. Kopfer (2001). WAP-LOG: Ein System zur mobilen Fahrzeugeinsatzsteuerung und Auftragsfortschrittkontrolle. In: Grünert, Sebastian: Logistik Management - Supply Chain Management und e-Business, Teubner Verlag, Stuttgart, pp. 293-303

[ErWa97] Ernst, M., D. Walpukis (1997). Telekommunikation und Verkehr, Verlag Franz Vahlen, München

[GrTh00] Gruhn, V., A. Thiel (1998). Komponentenmodelle, Addison Wesley, München

[JuLa01] Jung J., B. van Laak (2001). Flottenmanagementsysteme - Grundlegende Technologien, Funktionen und Marktüberblick. Universität Koblenz. Arbeitsbericht des Instituts für Wirtschaftsinformatik, Koblenz

[Lewa98] Lewandowski, S. (1998). Frameworks for Computer-Based Client/Server Computing. In: ACM Computing Surveys, Vol. 30, No. 1, ACM Press, pp. 3-27

[OrHe96] Orfali, R., D. Harkey, J. Edwards (1996). The Essential Client/Server Survival Guide. Wiley Publ.

[SiEK03] Siek, K., E. Erkens, H. Kopfer (2003). Marktübersicht über Systeme zur Fahrzeugkommunikation im Straßengüterverkehr. Submitted to: Logistik Management, 5. Jahrgang, Ausgabe 1, Germa Press Verlag GmbH, Hamburg

[Szyp98] Szyperski, C (1998). Component Software - Beyond Object-Oriented Programmung, Addison-Wesley, Reading, MA

[Tolk99] Tolksdorf, R. (1999) XML und darauf basierende Standards. In Informatik Spektrum, Band 22, Heft 6, Springer Verlag, Heidelberg, pp. 407-421

[Tran03] Euro Telematik AG (2003). TransWatch die Dispositions-Zentrale. http://www.eurotelematik.de/, Stand: 10.02.2003

[Voda01] Vodafone Passo GmbH (2001), http://www.passo.de/servlet/home, Stand: 10.02.2003 